\title{
ANALYTICAL MODEL OF THE DYNAMICS OF RAILWAY SLEEPER
}

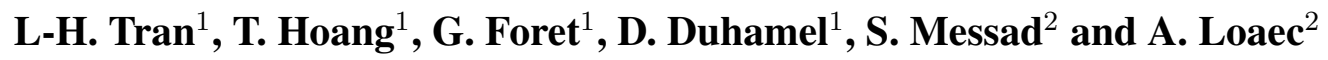 \\ ${ }^{1}$ Laboratoire Navier, UMR 8205, Ecole des Ponts, IFSTTAR, CNRS, \\ 6-8 Avenue Blaise Pascal, Cité Descartes, \\ Champs-sur-Marne, 77455 Marne-la-Vallée Cedex 2, France \\ e-mail: \{le-hung.tran, gilles.foret, denis.duhamel, tien.hoang\}@enpc.fr \\ ${ }^{2}$ SATEBA, 33 places des Corolles, 92400 Courbevoie, Courbevoie, France \\ e-mail: s.messad@sateba.com, arnaud.loaec@consolis.com
}

Keywords: Railway, analytical method, periodically supported beam, Fourier transform, Green function.

\begin{abstract}
The periodically supported beam is an analytical model of the dynamics of a railway track, where each rail together with its supports (sleepers) is independent of the other one. However, the sleepers connect the two rails and their behaviour could influence the response. This study develops an analytical model for this type of track by considering the sleepers as EulerBernoulli beams resting on a visco-elastic foundation. By using a relation between the reaction forces and displacement of the periodically supported beam [1], we can obtain a dynamic equation of a sleeper based on Dirac delta distribution. Then, the response of the sleepers can be obtained by using the Green function. This model is a fast method to calculate the dynamic responses of railway sleepers and track.
\end{abstract}




\section{Introduction}

Currently, there are many kinds of railway tracks constructed using different technologies. Among many choices of sleeper types, the concrete monoblock sleeper remains popular. In addition, the sleeper dynamic response is important because it affects the stability of the railway track and the maximum speed of the train. Substantial researches using analytic methods for rail track have been carried out, for example : the effect of wheel-rail contact forces $[17,18]$ or the vibration of the railway track (by considering the model of a ballasted track with discrete supports $[8,9,13,14,1]$ or the model of the infinite beam placed on a continuous foundation $[10,7,6,11])$. The studies of each component of the rail track were performed on the rail $[19,16,15]$, or on the ballast $[20,22]$.

An other important component of the railay track is the sleeper which has been investigated by using several different methods. The main objective is to analyse the sleeper behaviour and model it in the case of different moving charge values. The dynamic lateral resistance of the sleeper has been studied to better understand the interaction zones between the sleeper and the ballast layer [5]. Kaewunruen S. [23] researched the influence of the rail pad on the free vibration of the concrete sleeper by using finite element analysis. By using experimental and numerical methods, Laryea et al. [4] compared the sleeper's perfomance when made out of different materials. Some works focus on the pre-stressed concrete sleeper using FEM in 2D and in $3 \mathrm{D}[24,21]$.

In this paper, an analytical model is presented based on the theory of a beam resting on viscoelastic foundation. Indeed, the ratio between the length and the height of the sleeper $(L / h)$ is greater than 10 and we can consider the sleeper as an Euler-Bernoulli beam. The dynamic equation of the beam in the frequency domain by using the Fourier transform. Then by using the relation between the reaction forces and displacement of the beam [1] in the frequency domain and the Dirac delta distribution, the dynamic equation for the beam (sleeper) together with the rails and foundation is written and solved by using Green functions. The method of Green function to obtain the response of a beam structure to a moving mass has been successfully applied previously $[25,26]$. The use of Green function not only leads to exact results but also involves less cumbersome manipulations. Finally, the inverse Fourier transform gives the time responses of the beam.

The dynamic response of the sleeper has been investigated in two cases of moving charges. The numerical computations shows the displacements and reaction forces in the time domain. The model can be used for parametric analyses to study the influence of sleeper on the dynamic response. This simple model shows an efficient and fast way to estimate the responses of railway sleepers and can be further developed for the analysis of more complex cases.

\section{Formulations}

Let us consider a periodically supported beam subjected to moving forces as shown in Figure (1). In the steady-state, we suppose that all sleepers have the same response but with a delay which is equal to the time for the moving forces to cover the distance between two sleepers. By using this condition, Hoang et al. [1] have shown a relation between the beam displacement $\left(w_{r}\right)$ and reaction force $(R)$ at the sleeper position as follows :

$$
\hat{R}(\omega)=\mathcal{K}(\omega) \hat{w}_{r}(\omega)+\mathcal{Q}(\omega)
$$




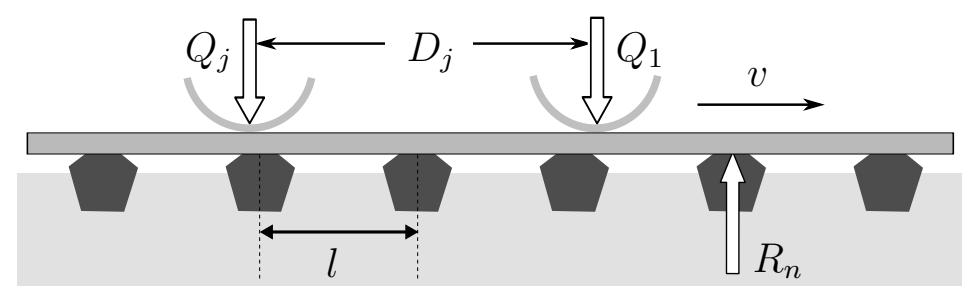

Figure 1: Periodically supported beam subjected to moving loads

where $\mathcal{K}(\omega)$ and $\mathcal{Q}(\omega)$ are so-called equivalent stiffness and pre-force of the periodically supported beam, calculated by :

$$
\begin{aligned}
& \mathcal{K}(\omega)=4 \lambda_{r}^{3} E_{r} I_{r}\left[\frac{\sin l \lambda_{r}}{\cos l \lambda_{r}-\cos \frac{\omega l}{v}}-\frac{\sinh l \lambda_{r}}{\cosh l \lambda_{r}-\cos \frac{\omega l}{v}}\right]^{-1} \\
& \mathcal{Q}(\omega)=\frac{\mathcal{K}(\omega)}{v E_{r} I_{r}\left[\left(\frac{\omega}{v}\right)^{4}-\lambda_{r}^{4}\right]} \sum_{j=1}^{K} Q_{j} \mathrm{e}^{-\mathrm{i} \omega \frac{D_{j}}{v}}
\end{aligned}
$$

where $\lambda_{r}=\sqrt[4]{\frac{\rho_{r} S_{r} \omega^{2}}{E_{r} I_{r}}}, \rho_{r}, E_{r}, S_{r}$ and $I_{r}$ are the density, Young's modulus, section and the longitudinal inertia of the rail respectively.

We now develop an analytical model for the dynamics of sleepers based on this equivalent system. Let us consider a railway track as shown in Figure 2. This track contains:

- Two rails: modelled by two periodically supported beams

- Rail pads: modelled by spring-damper systems

- Sleepers: modelled by Euler-Bernoulli's beams

- Ballast and foundation: modelled by a Kevin-Voight foundation

- Loads of train: concentrated moving loads

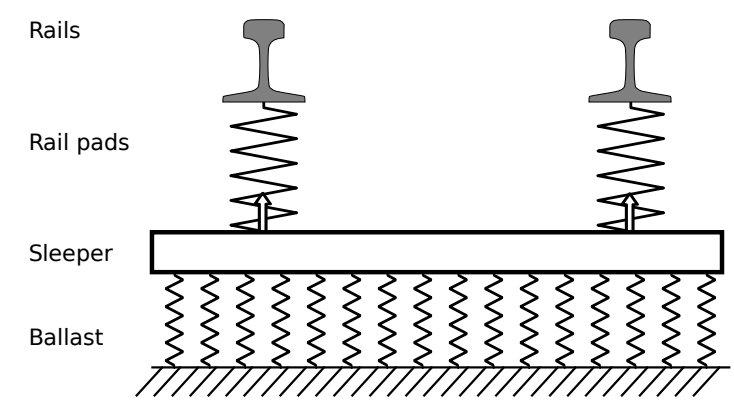

Figure 2: Dynamic model of a sleeper

The vertical displacement $w(x, t)$ of the sleeper under a force $F(x, t)$ is driven by the dynamic equation :

$$
E_{s} I_{s} \frac{\partial^{4} w(x, t)}{\partial x^{4}}+\rho_{s} S_{s} \frac{\partial^{2} w(x, t)}{\partial t^{2}}+k w(x, t)+\zeta \frac{\partial w(x, t)}{\partial t}=F(x, t)
$$


where $\rho_{s}, E_{s}, S_{s}$ and $I_{s}$ are the density, the Young's modulus, the section and the longitudinal inertia of the sleeper respectively; $k$ and $\zeta$ are the stiffness and the damping coefficient of the ballast layer.

The total force of two rails applied on the sleeper can be written with the help of Dirac functions as follows :

$$
F(x, t)=-R_{1}(t) \delta(x-a)-R_{2}(t) \delta(x+a)
$$

where $2 a$ is a distance between two rails, and $R_{1}, R_{2}$ are the reaction forces of the sleeper applied on two rails. These forces are driven by the equivalent systems of the two rails (equation (2)) as follows:

$$
\begin{aligned}
& \hat{R}_{1}=\mathcal{K} \hat{w}_{1}(\omega)+\mathcal{Q}_{1} \\
& \hat{R}_{2}=\mathcal{K} \hat{w}_{2}(\omega)+\mathcal{Q}_{2}
\end{aligned}
$$

In addition, the reaction forces $\hat{R}_{1}, \hat{R}_{2}$ can be expressed by the constitutive law of the two rail pads :

$$
\begin{aligned}
& \hat{R}_{1}=-k_{p}\left(\hat{w}_{1}(\omega)-\hat{w}(a, \omega)\right) \\
& \hat{R}_{2}=-k_{p}\left(\hat{w}_{2}(\omega)-\hat{w}(-a, \omega)\right)
\end{aligned}
$$

where $k_{p}=k_{r p}+\mathrm{i} \omega \zeta_{r p}$ are the dynamic stiffness of the rail pad $\left(k_{r p}, \zeta_{r p}\right.$ is the stiffness and damping coefficient of the rail pads) and $2 a$ is the track gauges. By substituting the aforementioned equation (5) into equation (6), we obtain

$$
\begin{aligned}
& \hat{R}_{1}=\frac{k_{p} \mathcal{K}}{k_{p}+\mathcal{K}} \hat{w}(a, \omega)+\frac{k_{p}}{k_{p}+\mathcal{K}} \mathcal{Q}_{1} \\
& \hat{R}_{2}=\frac{k_{p} \mathcal{K}}{k_{p}+\mathcal{K}} \hat{w}(-a, \omega)+\frac{k_{p}}{k_{p}+\mathcal{K}} \mathcal{Q}_{2}
\end{aligned}
$$

By combining equations (3) and (7), then by performing the Fourier transform, we obtain :

$$
E_{s} I_{s} \frac{\partial^{4} \hat{w}(x, \omega)}{\partial x^{4}}-\left(\rho S \omega^{2}-k_{b}\right) \hat{w}(x, \omega)=-\hat{R}_{1}(\omega) \delta(x-a)-\hat{R}_{2}(\omega) \delta(x+a)
$$

where $k_{b}=k+\mathrm{i} \zeta \omega$ is dynamic stiffness of the ballast.

Equation (8) describes the dynamics of the sleeper on the ballast. In order to solve these equations, we will use Green function for the dynamics of the beam defined by:

$$
\frac{\partial^{4} \mathrm{G}(x, a)}{\partial x^{4}}-\lambda_{s}^{4} \mathrm{G}(x, a)=\delta(x-a)
$$

where $\lambda_{s}=\sqrt[4]{\frac{\rho S \omega^{2}-k_{b}}{E_{s} I_{s}}}$. The general form of the Green function is :

$$
\mathrm{G}(x, a)= \begin{cases}A_{1} \cos \lambda_{s} x+A_{2} \sin \lambda_{s} x+A_{3} \cosh \lambda_{s} x+A_{4} \sinh \lambda_{s} x & \text { for } x \in[-L, a] \\ B_{1} \cos \lambda_{s} x+B_{2} \sin \lambda_{s} x+B_{3} \cosh \lambda_{s} x+B_{4} \sinh \lambda_{s} x & \text { for } x \in[a, L]\end{cases}
$$


where $2 L$ is the sleeper length. The boundary conditions of the Green function are :

$$
\begin{aligned}
& \mathrm{G}^{\prime \prime}(-L, a)=\mathrm{G}^{\prime \prime}(L, a)=\mathrm{G}^{\prime \prime \prime}(-L, a)=\mathrm{G}^{\prime \prime \prime}(L, a)=0 \\
& \mathrm{G}\left(a^{+}, a\right)-\mathrm{G}\left(a^{-}, a\right)=0 \\
& \mathrm{G}^{\prime}\left(a^{+}, a\right)-\mathrm{G}^{\prime}\left(a^{-}, a\right)=0 \\
& \mathrm{G}^{\prime \prime}\left(a^{+}, a\right)-\mathrm{G}^{\prime \prime}\left(a^{-}, a\right)=0 \\
& \mathrm{G}^{\prime \prime \prime}\left(a^{+}, a\right)-\mathrm{G}^{\prime \prime \prime}\left(a^{-}, a\right)=1
\end{aligned}
$$

where the prime $\left(^{\prime}\right)$ stands for the derivation with regard to $x$. By using this boundary condtion, we can obtain the expressions of $A_{i}, B_{i}(i=1,2,3,4)$ as shown in Appendix A.

The solution of equation (8) can be written with the help of the Green function as follows:

$$
\hat{w}(x, \omega)=\frac{-\hat{R}_{1}}{E_{s} I_{s}} \mathrm{G}(x, a)+\frac{-\hat{R}_{2}}{E_{s} I_{s}} \mathrm{G}(x,-a)
$$

In order to calculate $\hat{R}_{1}$ and $\hat{R}_{2}$, we substitute $x=a$ and $x=-a$ in the aforementioned equation and obtain:

$$
\left\{\begin{array}{l}
\hat{w}(a, \omega)=\frac{-\hat{R}_{1}}{E_{s} I_{s}} \mathrm{G}(a, a)+\frac{-\hat{R}_{2}}{E_{s} I_{s}} \mathrm{G}(a,-a) \\
\hat{w}(-a, \omega)=\frac{-\hat{R}_{1}}{E_{s} I_{s}} \mathrm{G}(-a, a)+\frac{-\hat{R}_{2}}{E_{s} I_{s}} \mathrm{G}(-a,-a)
\end{array}\right.
$$

By combining equations (7) and (13), we obtain

$$
\left\{\begin{array}{l}
\hat{R}_{1}=\kappa \frac{\mathcal{Q}_{1}[\mathrm{G}(-a,-a)+\chi]-\mathcal{Q}_{2} \mathrm{G}(a,-a)}{[\chi+\mathrm{G}(a, a)][\chi+\mathrm{G}(-a,-a)]-\mathrm{G}(-a, a) \mathrm{G}(a,-a)} \\
\hat{R}_{2}=\kappa \frac{\mathcal{Q}_{2}[\mathrm{G}(a, a)+\chi]-\mathcal{Q}_{1} \mathrm{G}(-a, a)}{[\chi+\mathrm{G}(a, a)][\chi+\mathrm{G}(-a,-a)]-\mathrm{G}(-a, a) \mathrm{G}(a,-a)}
\end{array}\right.
$$

where $\kappa=\frac{E_{s} I_{s}}{\mathcal{K}}$ and $\chi=E_{s} I_{s} \frac{k_{p}+\mathcal{K}}{k_{p} \mathcal{K}}$. Equation (14) defines the reaction force of the sleeper on the two rail. Then, the response of the sleeper can be obtained by using (12)

\section{Applications}

The dynamic response of the sleepers is calculated by using the formulae established in previous section for two cases : the same charges in both rails $\left(Q_{1}=Q_{2}\right)$ and the different charges in two rails $\left(Q_{1} \neq Q_{2}\right)$.

As the charge applied to the rail is periodic and moreover to simplify the comparison of results, the calculations are carried out in case of a point force. Some of the results in the case of a series of charges are shown in Appendix B. We consider a railway track parameters in Table 1.

Figure 3 shows the displacement of the sleeper in two cases: $Q_{1}=Q_{2}=100 \mathrm{kN}$ and $Q_{1}=100 \mathrm{kN}, Q_{2}=75 \mathrm{kN}$. The figures are obtained through the inverse Fourier transform of equation (12). The two rails positions are presented by two dash lines. The sleeper displacement in the two cases is minimum in the interval between two rails. In the first case, the minimum displacement is at the sleeper center $(x=0)$ and in the second case, it is $0.39 \mathrm{~mm}$ and this point is not the center. 


\begin{tabular}{llcl}
\hline Content & Unit & Notation & Value \\
\hline Young's modulus of the rail & $\mathrm{GPa}$ & $E_{r}$ & 210 \\
Moment of inertia of the rail & $\mathrm{m}^{4}$ & $I_{r}$ & $3.055 \mathrm{E}-5$ \\
Density of the rail & $\mathrm{kgm}^{-3}$ & $\rho_{r}$ & 7850 \\
Section of the rail & $\mathrm{m}^{2}$ & $s_{r}$ & $7.69 \mathrm{E}-3$ \\
Young's modulus of the sleeper & $\mathrm{GPa}$ & $E_{s}$ & 35 \\
Density of the sleeper & $\mathrm{kgm}^{-3}$ & $\rho_{s}$ & 2350 \\
Height of the sleeper & $\mathrm{m}$ & $h_{s}$ & 0.22 \\
Width of the sleeper & $\mathrm{m}$ & $w_{s}$ & 0.29 \\
Length of the sleeper & $\mathrm{m}$ & $2 L$ & 2.2245 \\
Track gauge & $\mathrm{m}$ & $2 a$ & 1.435 \\
Stiffness of the ballast & $\mathrm{MNm}^{-1}$ & $k$ & 158.11 \\
Damping coefficient of the ballast & $\mathrm{kNms}^{-1}$ & $\zeta$ & 41.30 \\
Stiffness of the rail pad & $\mathrm{MNm}^{-1}$ & $k_{r p}$ & 60 \\
Damping coefficient of rail pad & $\mathrm{kNms}^{-1}$ & $\zeta_{r p}$ & 77.76 \\
Train speed & $\mathrm{ms}^{-1}$ & $v$ & 44.4 \\
Charge on the first rail seat & $\mathrm{kN}^{-1}$ & $Q_{2}$ & 75 \\
Charge on the second rail seat & $\mathrm{kN}^{2}$ & $Q_{1}$ & 100 \\
Sleeper distance & $\mathrm{m}$ & $l$ & 0.6 \\
\hline
\end{tabular}

Table 1: Parameters of the analytical model

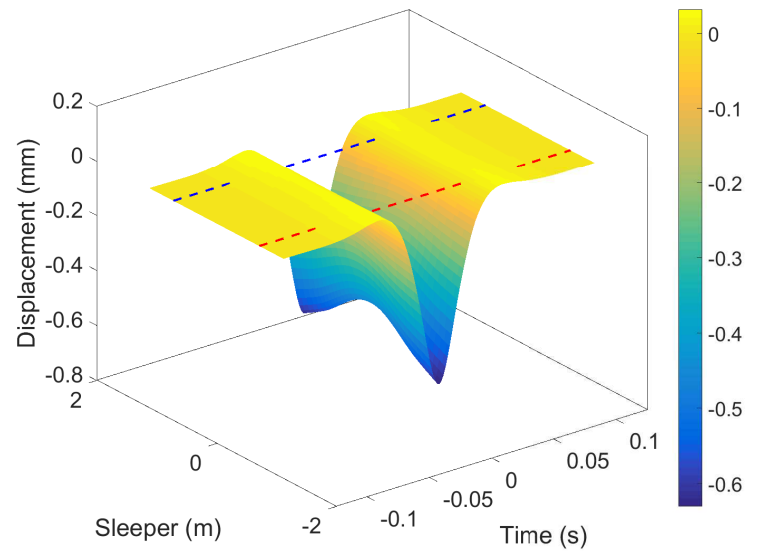

(a) $Q_{1}=Q_{2}=100 \mathrm{kN}$

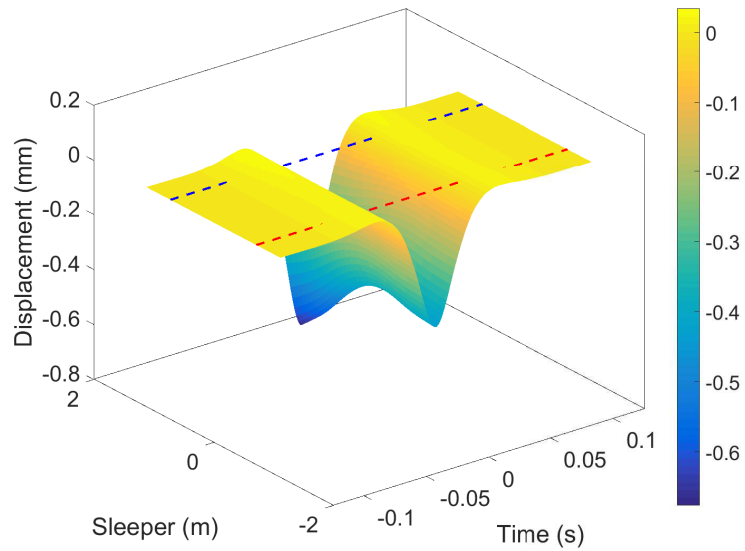

(b) $Q_{1}=100 \mathrm{kN}, Q_{2}=75 \mathrm{kN}$

Figure 3: Displacement of sleeper for the same loads and different loads on two rails

We observed this phenomenon because the displacement of the beam is not symmetric resulted by the different charges values. The sleeper displacements at the middle point and at the two rail seats are given by the Figure 4.

Figure $4 \mathrm{a}$ shows that the sleeper displacement at the rail seats are the same. The maximum value of the displacement at the rail seat is $0.57077 \mathrm{~mm}$. However, the displacements at the rail seats in Figure $4 \mathrm{~b}$ are not the same. Precisely, the first rail moves $0.41495 \mathrm{~mm}$ and the displacement of the second rail is $0.58390 \mathrm{~mm}$ (the red and blue curve). Moreover, Figure 5 shows the sleeper displacement in the two loading cases.

Under the action of the same forces, the deformation of the sleeper is symmetric and the 


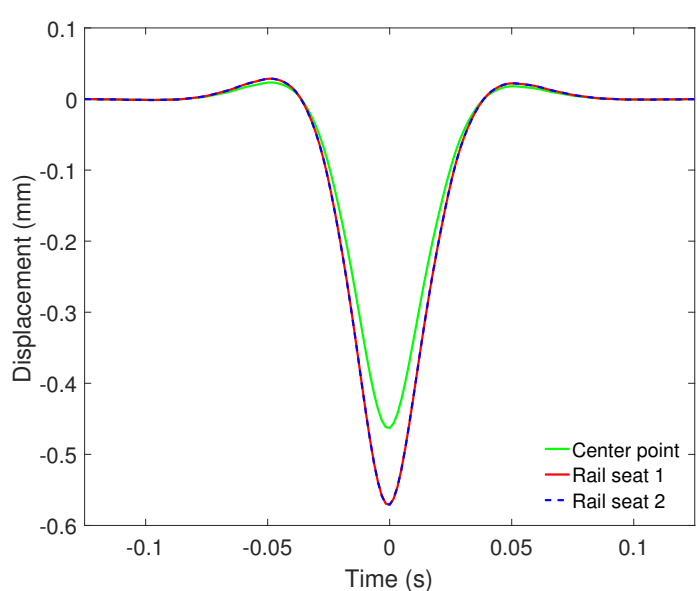

(a) $Q_{1}=Q_{2}=100 \mathrm{kN}$

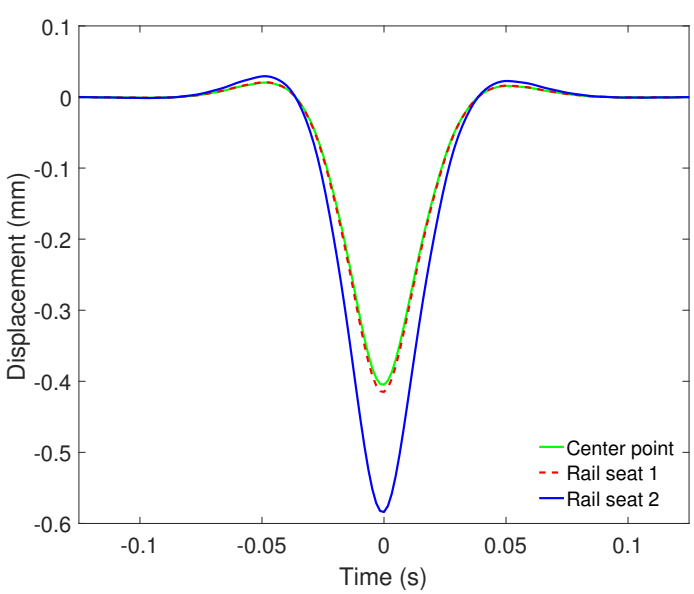

(b) $Q_{1}=100 \mathrm{kN}, Q_{2}=75 \mathrm{kN}$

Figure 4: Sleeper displacement under the moving charge at 3 positions : middle point (green curve), the first rail seat (red curve) and second rail seat (blue curve)

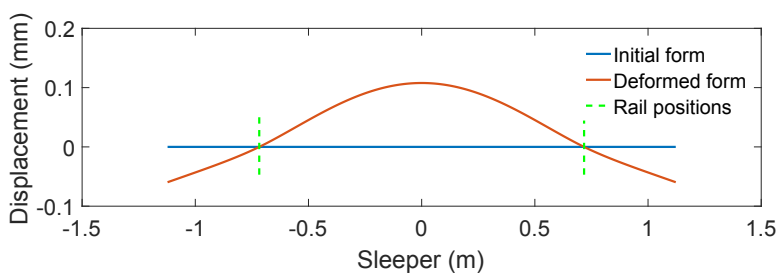

(a) $Q_{1}=Q_{2}=100 \mathrm{kN}$

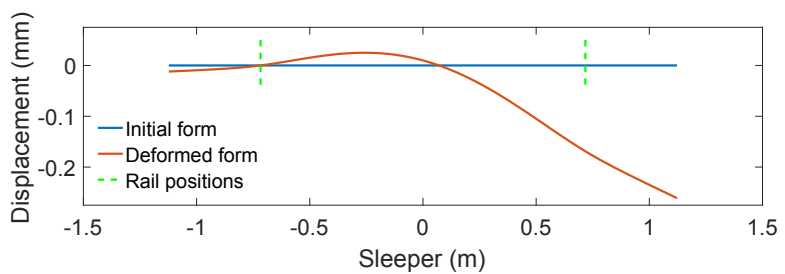

(b) $Q_{1}=100 \mathrm{kN}, Q_{2}=75 \mathrm{kN}$

Figure 5: Deformation form of the sleeper under the moving charge

displacements at the positions of two rail seats are at the same level. But in the second case, the displacements are different. The difference between two positions is referred to as the levelling of $0.17 \mathrm{~mm}$. This phenomenon shows that the sleeper is not only deformed but also tilted. When there is a signfication difference between the moving charges, the levelling will change the distance between two rails and influence the risk of derailment.

The reactions forces $\hat{R}_{1}$ and $\hat{R}_{2}$ are calculated by the equation (14). Obviously, the reaction forces at the rail seat $\hat{R}_{1}$ and $\hat{R}_{2}$ are equal when $Q_{1}=Q_{2}$. The reaction force is $42 \mathrm{kN}$ in this case.

When the two moving charges are different, the reaction force is bigger when the rail is subjected to the bigger charge ( $42 \mathrm{kN}$ and $31 \mathrm{kN}$ respectively). The damage of the wheels, rails etc., can lead to significantly different charges on the two rails.

\section{Conclusions}

In this study, an analytical model of the dynamics of railway sleeper has been investigated using the relation between the reaction force and the displacement in the frequency domain. The time responses are obtained by the inverse Fourier transform. The displacement at the critical sections (rail seats and center) has been evaluated for various moving charges. In the case of the imperfection of the moving charges, the phenomenon of levelling on the railway track occurs and it could influence the railway track stability. In future research, this model will be developed by using the Timoshenko beam in order to take in account of the sleeper rotation. Moreover, this 


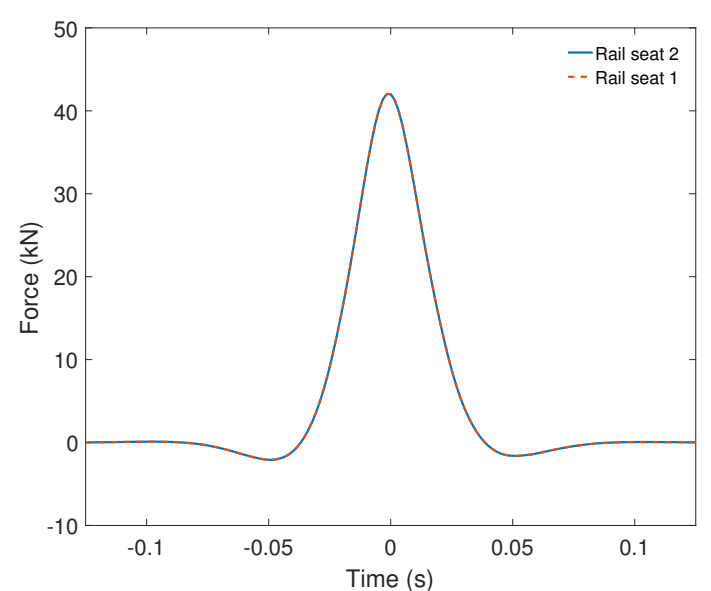

(a) $Q_{1}=Q_{2}=100 \mathrm{kN}$

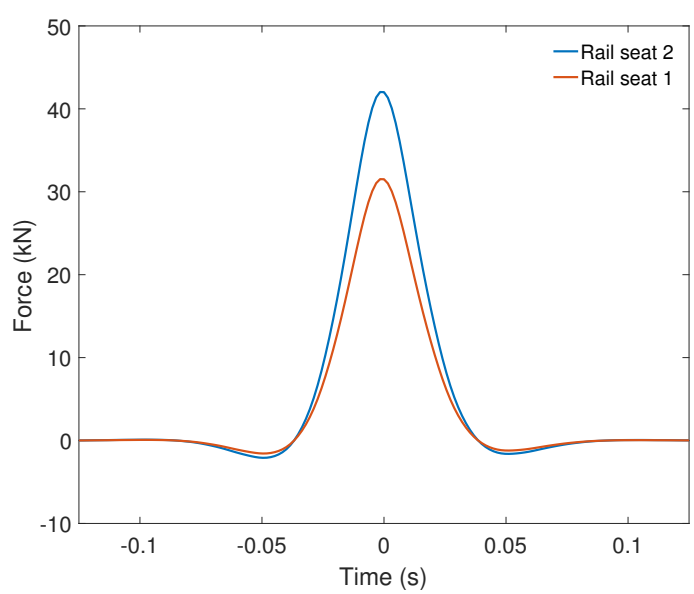

(b) $Q_{1}=100 \mathrm{kN}, Q_{2}=75 \mathrm{kN}$

Figure 6: Reaction force

model can be developed with other foundation behaviours such as a multi-layered foundation of ballast.

\section{A Calculation of Green function}

By substituting into equation (10), the first condition of the boundary condition in equation (11), we obtain can be rewritten as follows:

$$
\begin{aligned}
& -A_{1} \cos \lambda_{s} L+A_{2} \sin \lambda_{s} L+A_{3} \cosh \lambda_{s} L-A_{4} \sinh \lambda_{s} L=0 \\
& -A_{1} \sin \lambda_{s} L-A_{2} \cos \lambda_{s} L-A_{3} \sinh \lambda_{s} L+A_{4} \cosh \lambda_{s} L=0 \\
& -B_{1} \cos \left(\lambda_{s} L\right)-B_{2} \sin \left(\lambda_{s} L\right)+B_{3} \cosh \left(\lambda_{s} L\right)+B_{4} \sinh \lambda_{s} L=0 \\
& B_{1} \sin \left(\lambda_{s} L\right)-B_{2} \cos \left(\lambda_{s} L\right)+B_{3} \sinh \left(\lambda_{s} L\right)+B_{4} \cosh \lambda_{s} L=0
\end{aligned}
$$

We can also rewrite the four last conditions of equation (11) as follows:

$$
\begin{array}{r}
\left(B_{1}-A_{1}\right) \cos \lambda_{s} a+\left(B_{2}-A_{2}\right) \sin \lambda_{s} a+\left(B_{3}-A_{3}\right) \cosh \lambda_{s} a+\left(B_{4}-A_{4}\right) \sinh \lambda_{s} a=0 \\
-\left(B_{1}-A_{1}\right) \sin \lambda_{s} a+\left(B_{2}-A_{2}\right) \cos \lambda_{s} a+\left(B_{3}-A_{3}\right) \sinh \lambda_{s} a+\left(B_{4}-A_{4}\right) \cosh \lambda_{s} a=0 \\
-\left(B_{1}-A_{1}\right) \cos \lambda_{s} a-\left(B_{2}-A_{2}\right) \sin \lambda_{s} a+\left(B_{3}-A_{3}\right) \cosh \lambda_{s} a+\left(B_{4}-A_{4}\right) \sinh \lambda_{s} a=0 \\
\left(B_{1}-A_{1}\right) \sin \lambda_{s} a-\left(B_{2}-A_{2}\right) \cos \lambda_{s} a+\left(B_{3}-A_{3}\right) \sinh \lambda_{s} a+\left(B_{4}-A_{4}\right) \cosh \lambda_{s} a=\lambda_{s}^{-3}
\end{array}
$$

The aforementioned equation leads to the following result:

$$
\begin{array}{lll}
B_{1}-A_{1}=\frac{\sin \lambda_{s} a}{2 \lambda_{s}^{3}} & \text { and } & B_{2}-A_{2}=-\frac{\cos \lambda_{s} a}{2 \lambda_{s}^{3}} \\
B_{3}-A_{3}=-\frac{\sinh \lambda_{s} a}{2 \lambda_{s}^{3}} & \text { and } & B_{4}-A_{4}=\frac{\cosh \lambda_{s} a}{2 \lambda_{s}^{3}}
\end{array}
$$


By substituting equation (16) into equation (15), we obtain

$$
\begin{aligned}
& A_{1}=\frac{1}{4 \lambda_{s}^{3}} \frac{-\cosh \lambda_{s} L \cos \lambda_{s}(L-a)+\sinh \lambda_{s} L \sin \lambda_{s}(L-a)+\cosh \lambda_{s} a}{\cos \lambda_{s} L \sinh \lambda_{s} L+\sin \lambda_{s} L \cosh \lambda_{s} L} \\
& A_{2}=\frac{1}{4 \lambda_{s}^{3}} \frac{-\cosh \lambda_{s} L \sin \lambda_{s}(L-a)+\sinh \lambda_{s} L \cos \lambda_{s}(L-a)+\sinh \lambda_{s} a}{\cos \lambda_{s} L \sinh \lambda_{s} L-\sin \lambda_{s} L \cosh \lambda_{s} L} \\
& A_{3}=\frac{1}{4 \lambda_{s}^{3}} \frac{-\cos \lambda_{s} L \cosh \lambda_{s}(L-a)-\sin \lambda_{s} L \sinh \lambda_{s}(L-a)-\cos \lambda_{s} a}{\cos \lambda_{s} L \sinh \lambda_{s} L+\sin \lambda_{s} L \cosh \lambda_{s} L} \\
& A_{4}=\frac{1}{4 \lambda_{s}^{3}} \frac{\sin \lambda_{s} a+\sin \lambda_{s} L \cosh \lambda_{s}(L-a)-\cos \lambda_{s} L \sinh \lambda_{s}(L-a)}{\cos \lambda_{s} L \sinh \lambda_{s} L-\sin \lambda_{s} L \cosh \lambda_{s} L} \\
& B_{1}=\frac{1}{4 \lambda_{s}^{3}} \frac{-\cosh \lambda_{s} a+\sinh \lambda_{s} L \sin \lambda_{s}(L+a)-\cosh \lambda_{s} L \cos \lambda_{s}(L+a)}{\cos \lambda_{s} L \sinh \lambda_{s} L+\sin \lambda_{s} L \cosh \lambda_{s} L} \\
& B_{2}=\frac{1}{4 \lambda_{s}^{3}} \frac{\cosh \lambda_{s} L \sin \lambda_{s}(L+a)-\sinh \lambda_{s} L \cos \lambda_{s}(L+a)+\sinh \lambda_{s} a}{\cos \lambda_{s} L \sinh \lambda_{s} L-\sin \lambda_{s} L \cosh \lambda_{s} L} \\
& B_{3}=\frac{1}{4 \lambda_{s}^{3}} \frac{-\cos \lambda_{s} a-\sin \lambda_{s} L \sinh \lambda_{s}(L+a)-\cos \lambda_{s} L \cosh \lambda_{s}(L+a)}{\cos \lambda_{s} L \sinh \lambda_{s} L+\sin \lambda_{s} L \cosh \lambda_{s} L} \\
& B_{4}=\frac{1}{4 \lambda_{s}^{3}} \frac{-\sin \lambda_{s} a-\cos \lambda_{s} L \sinh \lambda_{s}(L+a)+\sin \lambda_{s} L \cosh \lambda_{s}(L+a)}{\cosh \lambda_{s} L \sin \lambda_{s} L-\sinh \lambda_{s} L \cos \lambda_{s} L}
\end{aligned}
$$

\section{B Example in the case of series of charges}

Here, we present an example with the series of charges on two rails. The distance between two wheels is $D=3(\mathrm{~m})$ and the length of the wagon is $H=18 \mathrm{~m}$. In this example, we have one imperfection at the wheel number 2 on the first rail with $\mathcal{Q}=75 \mathrm{kN}$ and the other moving charges $\mathcal{Q}=100 \mathrm{kN}$.

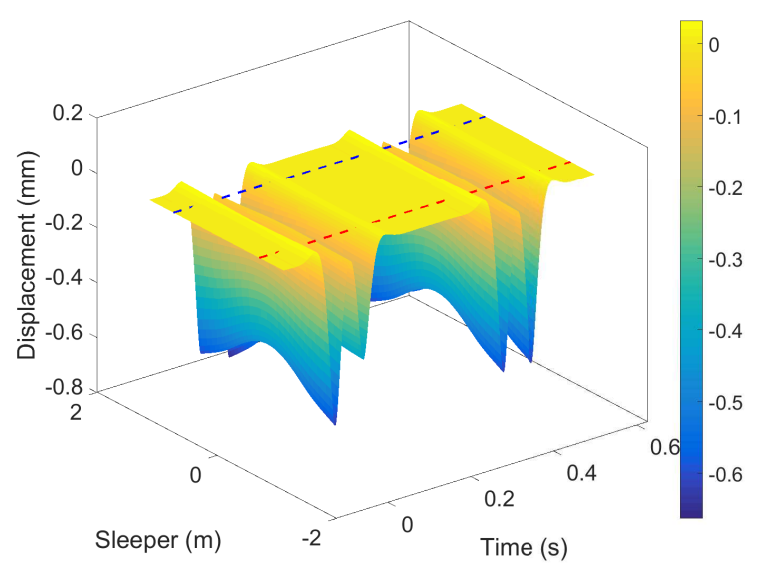

(a) Sleeper displacement

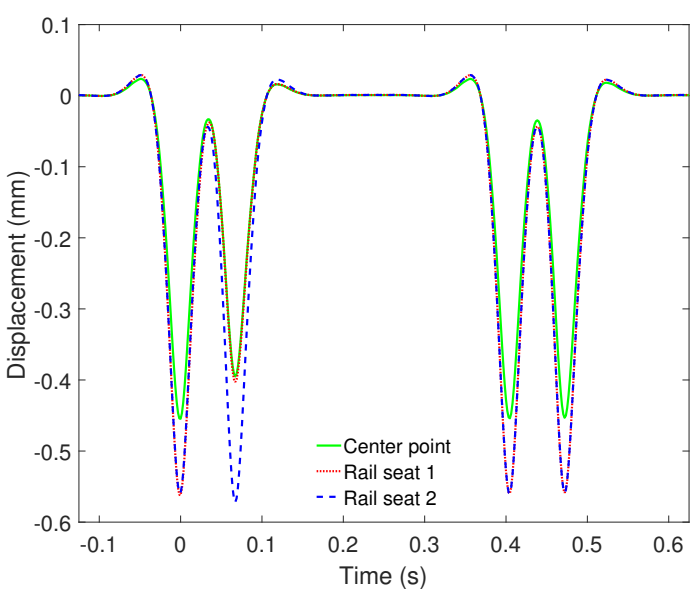

(b) Sleeper displacement at middle and two rail seats

Figure 7: Sleeper displacement 


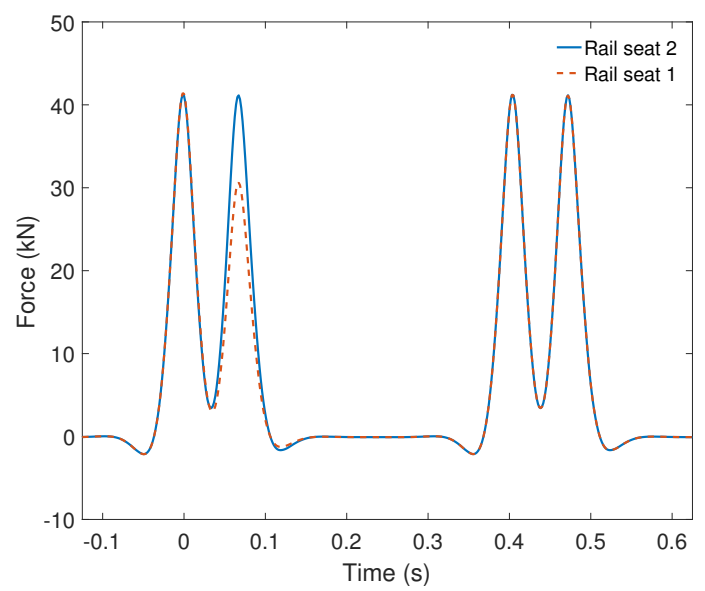

Figure 8: Reaction forces at two rail seats

\section{REFERENCES}

[1] T. Hoang, D. Duhamel, G. Foret, H.P. Yin, P. Joyez, R. Caby, Calculation of force distribution for a periodically supported beam subjected to moving loads. Journal of Sound and Vibration, 388, 327-338, 2017.

[2] S.L. Grassie, Dynamic modelling of concrete railway sleepers Journal of Sound and Vibration, 187, 799-813, 1995.

[3] C. Guigou-Carter, M. Villot, B. Guillerme, C. Petit, Analytical and experimental study of sleeper SAT S 312 in slab track Sateba system Journal of Sound and Vibration, 293, 878-887, 2006.

[4] S. Laryea, M. Safari-Baghsorkhi, J-F. Ferellec, G.R. McDowell, C. Chen, Comparison of performance of concrete and steel sleepers using experimental and discrete element methods Transportation Geotechnics, 1 225-240, 2014.

[5] M. Esmaeili, S.A.S. Hosseini, M. Sharavi, Experimental assessment of dynamic lateral resistance of railway concrete sleeper Soil Dynamics and Earthquake Engineering, 82 40-54, 2016.

[6] V.H. Nguyen, D. Duhamel, Finite element procedures for nonlinear structures in moving coordinates. Part II: Infinite beam under moving harmonic loads Computers \& Structures, 86 2056-2063, 2008.

[7] V.H. Nguyen, D. Duhamel, Finite element procedures for nonlinear structures in moving coordinates. Part 1: Infinite bar under moving axial loads Computers \& Structures, 84 1368-1380, 2006. pages $=1368-1380$,

[8] D.J. Mead, Y. Yaman, The response of infinite periodic beams to point harmonic forces: A flexural wave analysis Journal of Sound and Vibration, 144, 507-529, 1991.

[9] D.M. Mead, Wave propagation in continuous periodic structures : Research contributions from southamton, 1964-1995 Journal of Sound and Vibration, 190, 495-524, 1996. 
[10] L. Fryba Vibration of solids and structures under moving load. Thomas Telford, 1972.

[11] H. Ding, L-Q. Chen, S-P. Yang, Convergence of Galerkin truncation for dynamic response of finite beams on nonlinear foundations under a moving load Journal of Sound and Vibration, 331, 2426-2442, 2012.

[12] X. Sheng, C.J.C Jones, M. Petyt, Ground vibration generated by a harmonic load acting on a railway track Journal of Sound and Vibration, 225, 3-28, 1999.

[13] X. Sheng, C.J.C Jones, D.J. Thompson, Responses of infinite periodic structures to moving or stationary harmonic loads Journal of Sound and Vibration, 282, 125-149, 2005.

[14] X. Sheng, M. Li, C.J.C Jones, D.J. Thompson, Using the Fourier-series approach to study interactions between moving wheels and a periodically supported rail Journal of Sound and Vibration, 303, 873-894, 2007.

[15] D. Kostovasilis, D.J. Thompson, M.F.M. Hussein, A semi-analytical beam model for the vibration of railway tracks Journal of Sound and Vibration, 393, 312-337, 2017.

[16] D.J. Thompson, Experimental analysis of wave propagation in railway track Journal of Sound and Vibration, 203, 867-888, 1997.

[17] A. Matsumoto, Y. Sato, H. Ohno, M. Tomeoka, K. Matsumoto, J. Kurihara, T. Ogino, M. Tanimoto, Y. Kishimoto, Y. Sato, T. Nakai, A new measuring method of wheelrail contact forces and related considerations Wear, 265, 1518-1525, 2008.

[18] R. Bogacz, K. Frischmuth, On dynamic effects of wheelrail interaction in the case of Polygonalisation Mechanical Systems and Signal Processing, 79, 166-173, 2016.

[19] L. Gavrić, Computation of propagative waves in free rail using a finite element technique Journal of Sound and Vibration, 185, 531-543, 1995.

[20] G. Di Mino, M. Di Liberto, C. Maggiore, S. Noto, A Dynamic Model of Ballasted Rail Track with Bituminous Sub-Ballast Layer Procedia - Social and Behavioral Sciences, 53 366-378, 2012.

[21] A.A. Arab, S.S. Badie, M.T. Manzari, A methodological approach for finite element modeling of pretensioned concrete members at the release of pretensioning Engineering Structures, 33, 1918-1929, 2011.

[22] A. Aikawa, Dynamic characterisation of a ballast layer subject to traffic impact loads using three-dimensional sensing stones and a special sensing sleeper Construction and Building Materials, 92 23-30, 2015.

[23] S. Kaewunruen, A.M. Remennikov, Sensitivity analysis of free vibration characteristics of an in situ railway concrete sleeper to variations of rail pad parameters Journal of Sound and Vibration, 298, 453-461, 2006.

[24] G. Kumaran, D. Menon, K. Krishnan Nair, Dynamic studies of railtrack sleepers in a track structure system Journal of Sound and Vibration, 268, 485-501, 2003. 
[25] A.S. Mohamad, Tables of Green's functions for the theory of beam vibrations with general intermediate appendages International Journal of Solids and Structures, 31, 257-268, 1994.

[26] M.A. Foda, Z. Abduljabbar, A dynamic Green function formulation for the response of a beam structure to a moving mass Journal of Sound and Vibration, 210, 295-306, 1998. 